\title{
Leaking chemokines confuse neutrophils
}

\author{
Alex Marki ${ }^{1}$ and Klaus Ley \\ 'La Jolla Institute for Immunology, La Jolla, California, USA. ² Department of Bioengineering and ${ }^{3}$ Institute of Engineering in Medicine, UCSD, La Jolla, California, USA.
}

\begin{abstract}
The physical integrity of endothelial cells (ECs) lining the blood vessels regulates the inflammatory response. Both innate immunity and inflammatory disorders hinge on the EC-neutrophil interaction. Neutrophil binding, rolling, and migrating along and between ECs is associated with vascular permeability. In this issue of the $J C l$, Owen-Woods et al. tracked neutrophils in vivo in venules of mouse striated muscle and revealed how endothelial permeability can affect neutrophil trafficking. Strikingly, many neutrophils that migrated between EC junctions were able to rejoin the blood circulation. Further, the chemokine and neutrophil chemoattractant, CXCL1, drove this reverse transendothelial migration (rTEM). This paradigmshifting study provides a mechanism for distal organ damage as well as an explanation for sepsis-associated acute respiratory distress syndrome.
\end{abstract}

ma CXCL1 is sufficient to initiate neutrophil reverse transendothelial migration (rTEM) back into the lumen.

The authors applied cutting-edge intravital confocal microscopy of the cremaster muscle microvasculature. In this model the cremaster muscle was exteriorized, opened, and spread out for imaging while being superfused with a physiological buffer. This widely used model allows administration of compounds in the superfusion buffer while simultaneously observing transendothelial diffusion of tracer molecules and migration of neutrophils (8). Owen-Woods et al. developed a brilliant approach to label those neutrophils that visited the subendothelial space and then returned to the vessel lumen. They labeled the circulating neutrophils by injecting biotinylated anti-Ly6G antibody intravenously and superfused the exteriorized cremaster muscle with fluorescently labeled streptavidin that is retained in the extravascular space, presumably because it is too large $(\sim 55 \mathrm{kDa})$ to diffuse through a leaky vessel wall against the stream of extravasating blood plasma. As the biotinylated neutrophils entered the subendothelial space, they bound streptavidin and became fluorescently labeled. With this elegant method, the authors tracked the fate of rTEM neutrophils, which first accumulated in the lungs and later in the bone marrow. rTEM neutrophil counts in the lung correlated with leakiness of the lung vasculature, suggesting that rTEM neutrophils caused distal organ damage. Distal organ damage in response to injury, for example ischemia followed by reperfusion, has been known for decades, but this study offers a convincing mechanism by which this occurs.

\section{The pathomechanism of sepsis} The mechanism discovered by OwenWoods and colleagues may also contribute to the pathomechanism of sepsis. Based on the latest consensus definition, sepsis is diagnosed when proven or suspected bacterial or fungal infection and organ dysfunction are present $(9,10)$. For organ 
dysfunction assessment, the performance of the cardiovascular system, lungs, blood coagulation system, liver, kidneys, and central nervous system are scored (Sequential Organ Failure Assessment [SOFA] score) (10). Sepsis can progress into septic shock, a condition with even higher mortality. This happens when vascular permeability increases to the extent that the immense blood plasma extravasation results in blood pressure drop and consequential tissue hypoperfusion and hypoxia $(10,11)$. The most frequent directly sepsis-related causes of death are unresponsive hypotension and respiratory failure (12). The underlying cause of these clinical symptoms is a dysregulated immune response to infection. Pathogenand damage-associated molecular patterns trigger proinflammatory responses from immune and endothelial cells, vascular permeability increases, and leukocyte recruitment to the infected tissue begins. However, at the same time immunosuppressive mechanisms (such as endotoxin tolerance of innate immune cells, lymphocyte apoptosis and exhaustion) are initiated (11). These mechanisms lead to delayed pathogen clearance due to immune paralysis and host damage due to the persistent proinflammatory state (11).

The mechanism described by OwenWoods could exacerbate sepsis. The increased neutrophil rTEM rate could contribute to delayed pathogen clearance, because fewer neutrophils will engage with the infected site (increased permeability diverted $20 \%$ of the TEM neutrophils). Quick eradication of the pathogen is crucial in sepsis treatment. Every hour delay in antibiotic administration was found to increase progression to septic shock by $8 \%$ (13). As shown in the current publication, rTEM neutrophils contribute to lung dysfunction as they accumulate around leaky vessels, a sign of acute respiratory distress syndrome (ARDS). Diagnostic criteria for ARDS are sudden onset of hypo-oxygenation and lung edema that is not explained by cardiac failure (14). The underlying cause of these symptoms is disruption of the alveolar-capillary barrier.

The contribution of neutrophils to ARDS development is well recognized but incompletely understood (15). The recruited neutrophils become activated and release various proinflammatory and proteolytic compounds, which will result in an immense increase in vascular permeability. ARDS can result from lung infection by inhaled pathogens, when neutrophil recruitment is initiated by the pathogen-activated resident macrophages via chemokine release (16), and after extrapulmonary infection (17). The mechanisms of neutrophil recruitment differ greatly between organs. In the lung capillaries physical entrapment of the circulating neutrophils plays a dominant role, and the involvement of selectins and integrins is minor (1). Owen-Woods et al. found that rTEM neutrophils showed increased $\beta 1$ and $\beta 2$ integrin and ICAM- 1 expression on the cell surface, but it is unknown whether rTEM neutrophil accumulation in the lung is a consequence of upregulation of these adhesion molecules.

\section{Human relevance}

Owen-Woods and colleagues made their discovery in mice, whose immune system is known to differ from that of humans (18). Nevertheless, the human relevance of these findings is supported by the documented presence of increased vascular permeability (19), blood cytokine storm (increased IL-1 $\beta$, IL-6, IL-8, IL-10, TNF, and CCL-2) (20, 21), and neutrophil accumulation in ARDS (16). Additionally, in vitro experiments showed that human neutrophils following rTEM upregulate ICAM-1 expression similarly to the mouse neutrophils $(22,23)$.

This paradigm-changing study (7) raises several new questions. Does increased vascular permeability induce rTEM in every organ? Will all of these rTEM neutrophils home into the lung? Can rTEM neutrophil accumulation in the lung protect against secondary lung infection? Further studies are necessary to help us understand the relevance of this permeability-dependent rTEM mechanism for human diseases.

\section{Acknowledgments}

$\mathrm{KL}$ is supported by the NIH under award number HL078784. AM is supported by the Tullie and Rickey Families SPARK Award at the La Jolla Institute for Immunology.

Address correspondence to: Klaus Ley, La Jolla Institute for Immunology, 9420 Athena Circle Drive, La Jolla, California 92037, USA Phone: 858.752.6661; Email: klaus@lji.org.
1. Maas SL, Soehnlein O, Viola JR. Organ-specific mechanisms of transendothelial neutrophil migration in the lung, liver, kidney, and aorta. Front Immunol. 2018;9:2739.

2. Vestweber D. How leukocytes cross the vascular endothelium. Nat Rev Immunol. 2015;15(11):692-704.

3. Filewod NC, Lee WL. Inflammation without vascular leakage. Science fiction no longer? Am J Respir Crit Care Med. 2019;200(12):1472-1476.

4. Heemskerk N, et al. F-actin-rich contractile endothelial pores prevent vascular leakage during leukocyte diapedesis through local RhoA signalling. Nat Commun. 2016;7:10493.

5. Finsterbusch M, Voisin MB, Beyrau M, Williams TJ, Nourshargh S. Neutrophils recruited by chemoattractants in vivo induce microvascular plasma protein leakage through secretion of TNF. JExp Med. 2014;211(7):1307-1314.

6. Kenne E, et al. Neutrophils engage the kallikrein-kinin system to open up the endothelial barrier in acute inflammation. FASEB J. 2019;33(2):2599-2609.

7. Owen-Woods C, et al. Local microvascular leakage promotes trafficking of activated neutrophils to remote organs. JClin Invest. 2020;130(5):2301-2318.

8. Rius C, Sanz MJ. Intravital microscopy in the cremaster muscle microcirculation for endothelial dysfunction studies. Methods Mol Biol. 2015;1339:357-366.

9. Rhodes A, et al. Surviving sepsis campaign: International guidelines for management of sepsis and septic shock: 2016. Crit Care Med. 2017;45(3):486-552.

10. Singer M, et al. The third international consensus definitions for sepsis and septic shock (Sepsis-3). JAMA. 2016;315(8):801-810.

11. van der Poll T, van de Veerdonk FL, Scicluna BP, Netea MG. The immunopathology of sepsis and potential therapeutic targets. Nat Rev Immunol. 2017;17(7):407-420.

12. Moskowitz A, et al. Reasons for death in patients with sepsis and septic shock. J Crit Care. 2017;38:284-288.

13. Whiles BB, Deis AS, Simpson SQ. Increased time to initial antimicrobial administration is associated with progression to septic shock in severe sepsis patients. Crit Care Med. 2017;45(4):623-629.

14. ARDS Definition Task Force, et al. Acute respiratory distress syndrome: the Berlin definition. JAMA. 2012;307(23):2526-2533.

15. Williams AE, Chambers RC. The mercurial nature of neutrophils: still an enigma in ARDS? Am J Physiol Lung Cell Mol Physiol. 2014;306(3):L217-L230.

16. Englert JA, Bobba C, Baron RM. Integrating molecular pathogenesis and clinical translation in sepsis-induced acute respiratory distress syndrome. JCI Insight. 2019;4(2):e124061.

17. Scott BNV, Kubes P. Death to the neutrophil! A resolution for acute respiratory distress syndrome? Eur Respir J. 2018;52(2):1801274.

18. Masopust D, Sivula CP, Jameson SC. Of mice, dirty mice, and men: using mice to understand human immunology. J Immunol. 2017;199(2):383-388. 
19. Opal SM, van der Poll T. Endothelial barrier dysfunction in septic shock. JIntern Med. 2015;277(3):277-293.

20. Matsumoto H, et al. The clinical importance of a cytokine network in the acute phase of sepsis. Sci Rep. 2018;8(1):13995.
21. Weber GF, et al. Interleukin-3 amplifies acute inflammation and is a potential therapeutic target in sepsis. Science. 2015;347(6227):1260-1265.

22. Woodfin A, et al. The junctional adhesion molecule JAM-C regulates polarized transendothelial migration of neutrophils in vivo. Nat Immunol.
2011;12(8):761-769.

23. Buckley CD, et al. Identification of a phenotypically and functionally distinct population of long-lived neutrophils in a model of reverse endothelial migration.J Leukoc Biol. 2006;79(2):303-311. 